\title{
Avaliação dos registros das consultas de enfermagem em ginecologia
}

\section{Evaluation of the registrations of the nursing consultations in gynecology}

\section{La evaluación de los registros de las consultas de enfermería en ginecología}

\author{
Ana Luiza Santos de Carvalho', Rianna Nargilla Silva Nobre", Nilza Maria de Abreu Leitão"', \\ Camila Teixeira Moreira Vasconcelos ${ }^{\mathrm{IV}}$, Ana Karina Bezerra Pinheiro ${ }^{\mathrm{V}}$
}

\section{RESUMO}

A consulta de enfermagem ginecológica envolve um processo complexo, que requer entrevista e exame físico completos, a fim de subsidiar uma assistência de qualidade. $\mathrm{O}$ objetivo deste estudo foi avaliar registros de consultas de Enfermagem ginecológicas realizadas por acadêmicos de Enfermagem no Centro de Parto Natural da Universidade Federal do Ceará. Estudo retrospectivo, documental de abordagem predominantemente quantitativa, realizado em novembro de 2006, com uma amostra composta por 200 prontuários. O instrumento utilizado foi um check list, elaborado de acordo com os requisitos que o Ministério da Saúde preconiza para a consulta em ginecologia. Dos 42 itens pesquisados, apenas 3 foram registrados em $100 \%$ dos prontuários: nome, endereço e idade, e apenas 1 item não foi registrado em $100 \%$ dos casos: toque bimanual. Embora uma parcela dos dados ( 7 itens) tenha sido registrada em menos de $60 \%$ dos prontuários, o número de itens registrados em $100 \%$ deles foi ainda menor ( 3 itens). Esses resultados revelam a necessidade de acompanhamento dos acadêmicos de enfermagem pelos docentes no serviço de saúde, e a importância do registro de dados de forma completa e correta, de maneira a sanar as deficiências existentes e promover o preenchimento de $100 \%$ dos registros das consultas de enfermagem.

Palavras chave: Registros de enfermagem; Saúde da mulher; Educação em enfermagem; Ginecologia.

\section{ABSTRACT}

The gynecological nursing consultation involves a complex process of information, requiring the interview and physical examination completes, in order to subsidize an assistance of quality. The purpose of this study was to evaluate the records of the gynecological nursing consultation at the Center of Natural Childbirth of Federal University of Ceara. A retrospective documentary study with predominantly quantitative approach, held in November 2006, with a sample composed by 200 records. The instrument used was a check list, prepared in accordance with the requirements that the Ministry of Health recommended to be present in a consultation in gynaecology. Of the 42 items surveyed, only 3 were recorded in $100 \%$ of records: name, address and age, and only 1 item was not recorded in $100 \%$ of cases: bimanual touch. While a portion of the data ( 7 items) has been recorded in less than $60 \%$ of records, the number of items recorded in $100 \%$ of them was even lower ( 3 items). These results show the necessity to monitor the academics nursing faculty in the health service, and the importance of registration data in a comprehensive manner so as to correct and remedy existing weaknesses and promote the completion of $100 \%$ of the records of the nursing consultations.

Keywords: Records of nursing; Women's health; Education in nursing; Gynecology.

\section{RESUMEN}

La consulta de enfermería ginecológica implica un complejo proceso de información, lo que exige la entrevista y examen físico completa, con el fin de subvencionar una asistencia de calidad. El objetivo de este estudio fue evaluar los registros de las consultas de Enfermería de ginecología en el Centro de Parto Natural de la Universidad Federal de Ceará. Un estudio retrospectivo documental con enfoque

\footnotetext{
Acadêmica de Enfermagem da Universidade Federal do Ceará. Bolsista PIBIC/CNPq. Fortaleza, Ceará. E-mail: enflulu@hotmail.com

"Enfermeira pela Universidade Federal do Ceará. E-mail: rianninha_nsn@yahoo.com.br

"II Enfermeira pela Universidade Federal do Ceará. E-mail: nylzaleitao@yahoo.com.br

IV Enfermeira e mestranda da Universidade Federal do Ceará. E-mail: nylzaleitao@yahoo.com.br

$\checkmark$ Enfermeira e Professora Adjunto III do Departamento de Enfermagem. E-mail: anakarinaufc@hotmail.com
} 
predominantemente cuantitativos, que se celebró en noviembre de 2006, con una muestra compuesta por 200 documentos. El instrumento utilizado fue una lista de control, preparados de conformidad con los requisitos que el Ministerio de Salud recomienda que se presente en una consulta en ginecología. De los 42 temas estudiados, sólo 3 se registraron en el $100 \%$ de los registros: nombre, dirección y edad, y sólo el 1 artículo no se registró en el $100 \%$ de los casos: tacto bimanual. Si bien una parte de los datos ( 7 artículos) ha sido registrada en menos de $60 \%$ de los registros, el

\section{NTRODUÇÃO}

Dentre alguns desafios para se alcançar integralidade na assistência à saúde da mulher na Atenção Básica, estão as ações de controle dos cânceres de colo do útero e da mama. O câncer está entre as principais causas de morte na população feminina e, a mudança de hábitos, aliada ao estresse gerado pelo estilo de vida do mundo moderno, contribuem diretamente na incidência dessa doença. Alguns fatores como o tipo de alimentação, o sedentarismo, o tabagismo, a sobrecarga de responsabilidades aumento considerável do número de mulheres chefes de família - a competitividade, o assédio sexual e moral no mundo do trabalho, têm relevância destacada na mudança do perfil epidemiológico da situação e doença das mulheres $^{(1)}$.

O câncer de colo do útero (CCU) é o segundo mais comum entre mulheres no mundo. Anualmente são registrados cerca de 471 mil casos novos. Quase $80 \%$ deles ocorrem em países em desenvolvimento onde, em algumas regiões, é o câncer mais comum entre as mulheres. O número de casos novos de CCU esperados para o Brasil no ano de 2008 é de 18.680, com um risco estimado de 19 casos a cada 100 mil mulheres ${ }^{(2)}$.

Para gerar impacto sobre os múltiplos fatores que interferem nas ações de controle ao $\mathrm{CCU}$, é importante que a atenção às mulheres esteja pautada em uma equipe multiprofissional e com prática interdisciplinar. A interdisciplinaridade pressupõe, além das interfaces disciplinares tradicionais, a possibilidade da prática de um profissional se reconstruir na prática do outro(1). número de las partidas registradas en el $100 \%$ de ellos fue aún más baja ( 3 temas). Estos resultados muestran la necesidad de vigilar los académicos docentes de enfermería en el servicio de salud, y la importancia del registro de datos de manera global a fin de corregir y subsanar las deficiencias existentes y promover la realización del $100 \%$ de los registros de las consultas de enfermería.

Palabras clave: Los registros de enfermería; Salud de la mujer, Educación en enfermería; Ginecología.

Dentre as atribuições do enfermeiro na Atenção Básica, direcionadas à saúde da mulher, está realizar a consulta de enfermagem - coleta de exame preventivo e exame clínico das mamas, solicitar exames complementares e prescrever medicações, conforme protocolos ou outras normativas técnicas estabelecidas pelo gestor municipal, observadas as disposições legais da profissão - e realizar atividades de educação em saúde junto aos demais profissionais da equipe ${ }^{(1)}$.

O enfermeiro ao atuar nas ações de controle do CCU necessita realizar a consulta de enfermagem ginecológica, momento em que identifica aspectos da história de vida e saúde da cliente, faz orientações quanto à prevenção do câncer e das doenças sexualmente transmissíveis (DST), pode realizar visita domiciliária de acompanhamento aos casos de mulheres que tiveram que se submeter a conização, retiradas de nódulo de mama e outras atividades; como forma de contribuir para o envolvimento da família nos cuidados de saúde da cliente, bem como, resgatar o equilíbrio da dinâmica familiar e acompanhar a evolução do tratamento no domicílio(3).

A comunicação, a perspicácia e a disponibilidade de tempo são de fundamental importância, durante a consulta de enfermagem ginecológica, com o intuito de propiciar uma maior empatia e confiança entre profissional e cliente, além de minimizar a ansiedade, a timidez e a vergonha, contribuindo para abordagens que proponham a prevenção do câncer ginecológico ${ }^{(3)}$.

A consulta de enfermagem inicia-se com a entrevista, seguida do exame físico geral, 
exame ginecológico, colheita citológica, inspeção visual com ácido acético (IVA), teste de Schiller e toque bimanual. A anamnese da cliente, a identificação das diferentes vulnerabilidades e o exame físico devem constituir os principais elementos diagnósticos ${ }^{(4)}$.

A realização da consulta de enfermagem pressupõe necessariamente a aplicação do processo de enfermagem, que permite que o enfermeiro determine os diagnósticos de enfermagem e as intervenções de enfermagem correspondentes. A consulta de enfermagem é um procedimento da assistência desenvolvido com base em princípios ou pressupostos teórico-filosóficos definidos, empregando metodologia própria e que, em nosso meio, constitui a aplicação do processo de enfermagem $^{(5)}$. Dessa forma, segundo os mesmos autores, não podemos considerar a consulta como atendimentos ou orientações realizados em corredores.

O processo de Enfermagem consiste em cinco fases seqüenciais e inter-relacionadas: histórico, diagnóstico, planejamento, implementação e avaliação, as quais integram as funções intelectuais de solução de problemas, num esforço para definir as ações de Enfermagem ${ }^{(6)}$.

A aplicação do histórico de Enfermagem é feita durante toda a coleta de dados realizada pelo enfermeiro. Após este momento, segue-se à documentação no prontuário da cliente sobre todos os dados colhidos durante a entrevista e os dados que foram colhidos durante a realização do exame ginecológico ${ }^{(6)}$.

Observa-se a importância dos registros de Enfermagem, pois irão fornecer à equipe de saúde dados sobre a evolução do cliente, facilitar a comunicação entre a equipe de saúde, documentar as ações realizadas com o cliente, testemunhar as ações de Enfermagem em processos legais e fornecer subsídios para a conduta diagnóstica e terapêutica, servindo também para a avaliação dos cuidados de Enfermagem prestados e como fonte de aprendizagem $^{(7)}$.

É imprescindível durante o processo de formação do enfermeiro, a compreensão do seu papel na consulta ginecológica relacionado à problemática do câncer de colo do útero e de mama e das DST, bem como sobre a importância do registro adequado da consulta para que possa ser prestada uma assistência de qualidade à mulher. O compromisso em realizar um registro completo deve ser de todos os profissionais que utilizam o prontuário, bem como do acadêmico.

Em um serviço escola, a avaliação dos registros das consultas de enfermagem deve ser feita usualmente, haja vista os acadêmicos estarem em um processo de aprendizagem que requer monitoramento. $E$, nesse processo, os docentes também são responsáveis pela avaliação diária desses registros, tanto para avaliar se o registro está correto, como completo.

Diante desses fatos, a seguinte pergunta foi levantada: Como as consultas de enfermagem ginecológica do Centro de Parto Natural (CPN), que é um serviço escola, estão sendo registradas? Acredita-se que ao buscar essa resposta a pesquisa possa contribuir para o aperfeiçoamento dos registros de enfermagem, avaliar o processo de formação do acadêmico de enfermagem e prestar cuidados em ginecologia com qualidade.

Assim, o objetivo do presente estudo foi avaliar os registros das consultas de Enfermagem ginecológicas realizadas por acadêmicos de Enfermagem no Centro de Parto Natural (CPN).

\section{MÉTODOS}

Estudo retrospectivo, documental com abordagem predominantemente quantitativa, desenvolvido no Centro de Parto Natural Lígia Barros Costa, uma unidade de atenção primária de saúde, cujas atividades são coordenadas e desenvolvidas por enfermeiros e acadêmicos de enfermagem. Essa unidade está vinculada à Pró-Reitoria de Extensão da Universidade Federal do Ceará (UFC), sendo campo de prática para os acadêmicos de enfermagem.

Nesta instituição, a enfermagem atua junto à clientela (indivíduo, família e comunidade) mediante a realização de consultas de enfermagem nas diversas etapas do desenvolvimento da mulher (infância, adolescência, gravidez, puerpério e climatério). 
Carvalho ALS, Nobre RNS, Leitão NMA, Vasconcelos CTM, Pinheiro AKB. Avaliação dos registros das consultas de enfermagem em ginecologia. Revista Eletrônica de Enfermagem [Internet]. 2008;10(2):472-483. Available from: http://www.fen.ufg.br/revista/v10/n2/v10n2a18.htm

São realizadas consultas de pré-natal e ginecológicas, bem como visitas domiciliares as puérperas, gestantes faltosas ou mesmo quando as próprias clientes solicitam. Atualmente, é realizada busca ativa das clientes que não comparecem para receber o resultado dos exames preventivos.

$\mathrm{O}$ atendimento ginecológico conta com 02 (dois) consultórios, cujas consultas são realizadas no período da manhã por professores e acadêmicos da UFC. A clientela é composta por mulheres que buscam atendimentos ginecológicos, independentes da área à que pertencem.

Foi objeto de estudo o número total de consultas realizadas no primeiro semestre de 2006, totalizando 387 atendimentos. Os prontuários foram analisados e selecionados de acordo com os seguintes critérios de inclusão: possuir registro de consulta ginecológica com mulheres que já iniciaram atividade sexual e registro da realização do exame preventivo no primeiro semestre de 2006. Esses critérios foram estabelecidos, pois algumas mulheres chegaram a realizar entrevista, mas estavam impossibilitadas de realizar o exame preventivo por apresentarem alguma contra-indicação no momento (menstruação, relação sexual com menos de 24 horas ou outra intercorrência) e, foram excluídas as mulheres que não iniciaram a relação sexual, pois as mesmas não realizam o exame citopatológico. Após a aplicação dos critérios estabelecidos, obteve-se uma amostra de 200 prontuários.

A coleta de dados foi realizada no mês de Novembro de 2006, por meio de um check-list, elaborado mediante os requisitos que 0 Ministério da Saúde do Brasil ${ }^{(8)}$ preconiza para serem abordados em uma consulta em ginecologia. No check-list foram abordadas as seguintes variáveis: anamnese, queixas e duração, antecedentes pessoais, familiares, ginecológicos, obstétricos e sexuais, além do exame físico geral, exame das mamas, vulva e períneo, exame especular, inspeção visual com ácido acético (IVA), teste de Schiller e toque bimanual.

Os dados foram organizados no SPSS (Statistical Package for Social Sciences) versão
11.0. Após a organização dos dados, procedeuse a análise estatística descritiva.

O estudo respeitou os aspectos éticos e legais da Resolução 196/96 e faz parte do projeto Prevalência e Análise das lesões de mulheres portadoras de HPV que foi aprovado pelo comitê de Ética e Pesquisa da Universidade Federal do Ceará com parecer $n^{\circ}$ 315/05.

\section{RESULTADOS E DISCUSSÃO}

\section{Dados de I dentificação}

No presente estudo, $100 \%$ dos dados de identificação referentes ao nome, a idade e ao endereço analisados foram registrados (Tabela 1). Segundo estudo realizado por Carrara e colaboradores $^{(9)}$ a identificação da cliente deve ser feita da forma mais precisa possível, evitando erros e confusões que poderão ter conseqüências irreparáveis, pois quando estão incompletos ou preenchidos de forma incorreta podem gerar duplicidade ou a não identificação da cliente no momento da entrega do laudo. Ainda pode oferecer problema quando há necessidade de uma busca ativa, devido a um resultado que requer intervenção imediata ou pelo não retorno da cliente ao serviço. Além disso, a idade merece especial atenção, pela sua importância como fator de risco, pois certas afecções ginecológicas são mais comuns em determinados grupos etários ${ }^{(10)}$, como é o caso do $\mathrm{CCU}$, que atinge mais freqüentemente as mulheres com mais de $40 \operatorname{anos}^{(11)}$. 
Tabela 1: Distribuição segundo os registros dos dados de identificação da Casa de Parto Natural. Fortaleza, 2006.

\begin{tabular}{|c|c|c|}
\hline VARIÁVEIS & $\mathbf{N}^{\circ}$ & $\%$ \\
\hline \multicolumn{3}{|l|}{ NOME } \\
\hline Sim & 200 & 100 \\
\hline Não & 0 & 0 \\
\hline \multicolumn{3}{|l|}{ ENDEREÇO } \\
\hline Sim & 200 & 100 \\
\hline Não & 0 & 0 \\
\hline \multicolumn{3}{|l|}{ I DADE } \\
\hline Sim & 200 & 100 \\
\hline Não & 0 & 0 \\
\hline \multicolumn{3}{|l|}{ ESCOLARI DADE } \\
\hline Sim & 94 & 46 \\
\hline Não & 106 & 53 \\
\hline \multicolumn{3}{|l|}{ ESTADO CI VI L } \\
\hline Sim & 67 & 34 \\
\hline Não & 133 & 66 \\
\hline
\end{tabular}

Em relação ao registro do estado civil e ao nível de escolaridade, verificou-se que a maioria dos acadêmicos $66 \%$ e $53 \%$ respectivamente, não registrou esses dois dados. O estado civil da cliente deve ser registrado, com o nome do parceiro para possível contato e informações sobre o estado de saúde da cliente $^{(9)}$. A escolaridade é um dado importante para registro, pois o enfermeiro deve sempre garantir que suas orientações fornecidas durante a consulta sejam acessíveis à clientela, bem como devido à associação entre a baixa escolaridade e a ocorrência de DST ${ }^{(11)}$. Assim, a importância de registrar o nível de escolaridade da cliente reflete na adequada intervenção que será realizada a fim de se obter os resultados esperados.

É importante também destacar que mulheres com déficit cultural e econômico são mais vulneráveis a contrair DST por não possuir habilidade em conversar e negociar o uso da camisinha com o parceiro. As que apresentam grau de instrução de mais anos de estudo, provavelmente são mais conscientes de seus direitos e têm mais poder para exigir sexo

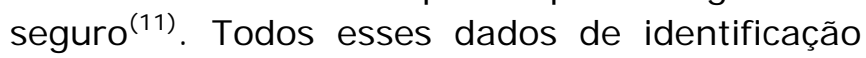
obtidos na consulta ginecológica são importantes ferramentas para que o enfermeiro desenvolva estratégias de prevenção do CCU através de atividades educativas individuais ou grupais, visto que representam o perfil da clientela e possíveis fatores de risco para essa doença.

O CCU é um problema de saúde pública devido às suas altas taxas de prevalência e mortalidade em mulheres de nível social e econômico baixos e em fase produtiva de suas vidas, indicando uma forte associação deste tipo de câncer com as condições de vida precárias, os baixos índices de desenvolvimento humano, a ausência ou fragilidade das estratégias de educação comunitária (promoção e prevenção em saúde) e com a dificuldade de acesso a serviços públicos de saúde para o diagnóstico precoce e o tratamento das lesões precursoras $^{(11)}$.

\section{Queixas}

Com relação às queixas e sua duração, verifica-se que somente a metade (50\%) registrou a queixa e, menos da metade $(46 \%)$, registrou há quanto tempo estes sintomas ocorrem. Em uma pesquisa realizada no interior do Ceará em um serviço de prevenção do CCU com 141 mulheres foi demonstrado que o motivo que mais se sobressaiu, com $56,7 \%$ das citações, para a busca da consulta a fim de realizar o exame citopatológico foi apresentar alguma queixa ${ }^{(12)}$. Nesse item da anamnese, deve-se anotar o motivo que levou a cliente a procurar o serviço de saúde e o tempo decorrido desde o aparecimento da doença, ou melhor, da sintomatologia atual. A queixa deve ser registrada, na medida do possível, com as próprias expressões usadas pela cliente ${ }^{(1)}$. As queixas ginecológicas relacionadas ao trato genital inferior constituem síndromes clínicas que não só devem ser valorizadas, mas sempre que possível, solucionadas antes da coleta do exame preventivo. É importante lembrar que a 
Carvalho ALS, Nobre RNS, Leitão NMA, Vasconcelos CTM, Pinheiro AKB. Avaliação dos registros das consultas de enfermagem em ginecologia. Revista Eletrônica de Enfermagem [Internet]. 2008;10(2):472-483. Available from: http://www.fen.ufg. br/revista/v10/n2/v10n2a18.htm

presença de colpites, leucorréias ou colpocervicites pode comprometer a interpretação da citopatologia. Nesses casos, a mulher deve ser tratada e retornar para a coleta $^{(1)}$. Dessa forma, esses dados revelaram certa deficiência neste item da entrevista que pode ser devido à dificuldade por parte do acadêmico em abordar a cliente ou devido ausência de queixa no momento da consulta. Contudo, a ausência da queixa também deveria ser registrada para que os dados estejam completos no prontuário e para, numa próxima consulta, saber se a queixa que a mulher apresenta é recorrente ou não.

\section{Antecedentes familiares e pessoais}

A pesquisa dos antecedentes familiares é de extrema importância em ginecologia, haja vista algumas doenças terem um forte componente hereditário, o que o enfermeiro precisa estar atento para oferecer meios de prevenir ou retardar o surgimento das mesmas, quando possível, ou detectá-las precocemente. Dentre os antecedentes familiares pesquisados neste estudo, destacaram-se: hipertensão arterial, diabetes mellitus e neoplasias.

Neste estudo, foi registrada a história familiar de hipertensão em $94 \%$ da amostra, a de diabetes mellitus e a de neoplasias em $92 \%$. A hipertensão arterial e o diabetes mellitus são doenças crônicas não transmissíveis, altamente prevalentes, de alto custo social e grande impacto na morbimortalidade da população brasileira e do mundo(13). A possibilidade de associação das duas doenças é da ordem de $50 \%$, o que requer, na grande maioria dos casos, o manejo das duas doenças num mesmo cliente. Além disso, essas duas doenças têm um forte componente hereditário(14) e, por isso, devem ser pesquisadas durante a anamnese. As taxas dessas doenças neste estudo foram altas, o que revelou a preocupação dos acadêmicos de enfermagem em pesquisar o histórico familiar das clientes com vistas a fornecer subsídios à formulação de hipóteses diagnósticas e/ou realização de orientações educativas. Uma hipótese que pode ser aventada para não se ter atingido os $100 \%$, é que algumas mulheres perderam os pais muito cedo, ou são adotadas, ou mesmo não moram com os pais, o que as impede de responder a estes itens.

Em relação às neoplasias familiares, devem ser pesquisadas principalmente as neoplasias ginecológicas (útero, ovário e endométrio) e câncer de mama ${ }^{(10)}$. A neoplasia maligna ovariana, apesar de rara, é a primeira causa de morte entre as neoplasias ginecológicas. São consideradas de alto risco, mulheres com um ou mais familiares de primeiro grau com história de carcinoma de ovário na pré-menopausa ou com um familiar de primeiro grau e um ou mais familiares de segundo $\operatorname{grau}^{(10)}$. Existem famílias com síndromes neoplásicas em associação com o carcinoma ovariano. Algumas delas são: carcinoma de mama e ovário e o carcinoma de colo, mama e ovário(10).

Ao se investigar a história pessoal de DST, hipertensão, diabetes e neoplasias na maioria dos prontuários $(74,5 \%),(89,0 \%),(92,0 \%)$ e $(87,0 \%)$, respectivamente, estava registrada de maneira completa. A investigação da história pessoal de hipertensão, diabetes e neoplasias é importante devido à restrição de algumas medicações que podem ser usadas pelas mulheres e por serem fatores de risco para outras doenças.

Um exemplo disso é a terapia de reposição hormonal (TRH) que alivia os desconfortos da menopausa, porém deve ser avaliada com cautela para mulheres com suspeita ou com diagnóstico de câncer de mama, de útero ou de ovário, hipertensão, doenças cardíacas, dislipidemia ou arteriosclerose, diabetes e antecedentes familiares de anemia perniciosa ${ }^{(15)}$. Em relação ao aconselhamento anticoncepcional entre mulheres diabéticas ou não-diabéticas não há diferenças. Deve-se, porém, usar somente contraceptivos hormonais de baixo teor estrogênico, levando em conta seus efeitos deletérios sobre o controle glicêmico, a evolução da microangiopatia, o surgimento de hipertensão e a incidência de tromboembolismos. Na presença de qualquer destes fatores, usar preparações puramente progestacionais ou métodos de barreira, ou considerar a hipótese de realizar ligadura tubária ${ }^{(14)}$. Além disso, o diabetes está entre os fatores predisponentes para 
desencadeamento da candidíase, que é uma infecção fúngica cuja queixa principal é a leucorréia branca em grumos acompanhada ou não de prurido vulvar e/ou vaginal ${ }^{(10)}$.

\section{Antecedentes ginecológicos}

A idade da primeira menstruação (menarca) foi registrada em quase a totalidade da amostra analisada $(99,0 \%)$. Em relação às características dos ciclos menstruais, observase que, na maioria da amostra, foram registrados os aspectos de: duração $(72,5 \%)$, características do fluxo $(66,5 \%)$ e regularidade $(90,5 \%)$. O intervalo entre as menstruações deve ser, em média, de 28 dias, porém variações de 26 a 32 dias podem ser normais. A duração do fluxo menstrual é de 4 a 5 dias. Pequenas variações ( 3 a 8 dias) podem ocorrer e, também, são consideradas normais. A quantidade de sangue perdido deve oscilar entre 25 e $100 \mathrm{ml}$. As mulheres que sangram por 8 dias ou mais podem perder maior volume (até $200 \mathrm{ml}$ ). A cor deve ser vermelha escura, o conteúdo fluido, e não formar coágulos ${ }^{(16)}$.

A data da última menstruação (DUM) foi lembrada em $92,0 \%$ da amostra. O uso de métodos anticoncepcionais foi investigado em $89 \%$ da amostra estudada, porém $55 \%$ dos acadêmicos não anotaram há quanto tempo esta mulher usa o método. A DUM deve sempre ser verificada principalmente por dois motivos: para que possa ser afastada a hipótese de gravidez, para diferenciar um sangramento transvaginal anormal de menstruação, caso a mulher sangre no momento do exame.

Em relação à documentação sobre as Doenças Sexualmente Transmissíveis (DST), verificou-se que a maioria $(72,5 \%)$ havia registrado a ocorrência anterior de DST. As DST representam um sério impacto na saúde reprodutiva porque podem causar esterilidade, doença inflamatória pélvica, câncer de colo uterino, gravidez ectópica, infecções puerperais e recém-nascidos com baixo peso, além de interferir negativamente sobre à auto-estima ${ }^{(17)}$.

A data da realização do último exame preventivo foi registrada em $83,5 \%$ da amostra estudada, porém o resultado desse último exame não foi registrado em $81,5 \%$ dos prontuários. Esse dado torna-se relevante à medida que os exames citopatológicos servem com screening para o câncer de colo uterino e precisam ser monitorados para que haja um seguimento. Existem algumas possíveis explicações para este dado, como por exemplo: ou a mulher está procurando o serviço em questão pela primeira vez, e, por ter realizado o exame em outra instituição, não atentou para o fato de ser importante levar o exame anterior na consulta atual; ou a mulher não recebeu o resultado do exame anterior e, por isso não sabe referir o seu resultado; ou, por simples esquecimento da mulher ou do profissional.

Tendo em vista esse aspecto, é importante que o profissional que está prestando 0 atendimento notifique o motivo pelo qual não consta o resultado do exame, pois, dessa forma, o profissional fica isento em relação ao subregistro deste dado.

\section{Antecedentes obstétricos}

Em relação ao passado obstétrico, mais especificamente sobre os registros dos números de gestações, partos, tipos de partos, abortos, tipo de abortos, foram registrados respectivamente $(93 \%),(92,5 \%), \quad(87,5 \%)$, $(92 \%),(67,5 \%)$ (Tabela 2$)$. 
Tabela 2: Distribuição dos registros segundo antecedentes obstétricos da Casa de Parto Natural. Fortaleza, 2006.

\begin{tabular}{lcc}
\hline \multicolumn{1}{c}{ VARIÁVEIS } & $\mathbf{N}$ & $\%$ \\
\hline No GESTAÇÕES & \multicolumn{1}{c}{} \\
Sim & 186 & 93,0 \\
Não & 14 & 7,0 \\
No PARTOS & 185 & 92,5 \\
Sim & 15 & 7,5 \\
Não & & 87,5 \\
TI POS DE PARTOS & 176 & 12,5 \\
Sim & 24 & 92,0 \\
Não & & 8,0 \\
No ABORTOS & 184 & 67,5 \\
Sim & 16 & 32,5 \\
Não & & \\
TI POS DE ABORTOS & 135 & 65 \\
Sim & & \\
Não & &
\end{tabular}

As mulheres estão em risco de problemas relacionados ao sistema reprodutivo desde a idade da menarca até a menopausa e a idade mais avançada. Esses problemas englobam os distúrbios estruturais do útero e da vagina relacionados ao relaxamento pélvico e à incontinência urinária. Eles podem ser um resultado posterior do(s) parto(s). Por exemplo, as estruturas e os tecidos moles da vagina e da bexiga podem ser lesados durante o trabalho de parto prolongado, ou quando ocorre a desproporção céfalo-pélvica ${ }^{(16)}$. Para uma adequada avaliação do assoalho pélvico durante a consulta ginecológica, deve-se fazer uma história clínica detalhada da sintomatologia da cliente, uma avaliação específica das alterações pélvicas e verificar se há ou não uma correlação entre a história e os achados físicos. Para isso, é imprescindível levantar o passado obstétrico da mulher ${ }^{(10)}$.

No que se refere ao aborto, sabe-se que é uma prática ilegal na maior parte dos países da América Latina. No Brasil, é proibido, salvo em duas situações: feto concebido como resultado de estupro e gravidez que acarreta risco de vida para a mulher ${ }^{(18)}$. Talvez, por esse assunto ser polêmico, tenha havido uma certa dificuldade entre os acadêmicos em questionar o tipo de aborto realizado pela mulher.

\section{Antecedentes sexuais}

Em relação ao início da atividade sexual, dispareunia, número de parceiros, hábitos sexuais e sangramento pós-coito verificou-se que respectivamente, $96,0 \%, 73,5 \%, 90,0 \%$,
$66,0 \%$ e $74,5 \%$ dos dados foram preenchidos corretamente (Tabela 3). 
Carvalho ALS, Nobre RNS, Leitão NMA, Vasconcelos CTM, Pinheiro AKB. Avaliação dos registros das consultas de enfermagem em ginecologia. Revista Eletrônica de Enfermagem [Internet]. 2008;10(2):472-483. Available from: http://www.fen.ufg.br/revista/v10/n2/v10n2a18.htm

Tabela 3: Distribuição dos registros segundo antecedentes sexuais. Casa de Parto Natural. Fortaleza, 2006.

\begin{tabular}{|c|c|c|}
\hline VARIÁVEIS & $\mathbf{N}$ & $\%$ \\
\hline \multicolumn{3}{|l|}{ I NÍ CI O DE ATI VI DADE SEXUAL } \\
\hline Sim & 192 & 96,0 \\
\hline Não & 8 & 4,0 \\
\hline \multicolumn{3}{|l|}{ LI BIDO } \\
\hline Sim & 35 & 17,5 \\
\hline Não & 165 & 82,5 \\
\hline \multicolumn{3}{|l|}{ ORGASMO } \\
\hline Sim & 168 & 82,0 \\
\hline Não & 36 & 18,0 \\
\hline \multicolumn{3}{|l|}{ DI SPAREUNIA } \\
\hline Sim & 147 & 73,5 \\
\hline Não & 53 & 26,5 \\
\hline \multicolumn{3}{|l|}{ No PARCEI ROS } \\
\hline Sim & 180 & 90,0 \\
\hline Não & 20 & 10,0 \\
\hline \multicolumn{3}{|l|}{ HÁBITOS SEXUAIS } \\
\hline Sim & 132 & 66,0 \\
\hline Não & 68 & 34,0 \\
\hline \multicolumn{3}{|l|}{ SANGRAMENTO PÓS-COITO } \\
\hline Sim & 149 & 74,5 \\
\hline Não & 51 & 25,5 \\
\hline
\end{tabular}

Esses dados são importantes, pois existe uma associação entre o câncer do colo do útero e a atividade sexual das mulheres, com ocorrência elevada naquelas com múltiplos parceiros sexuais e/ou que iniciaram a atividade sexual precocemente, que tiveram muitos filhos ou que seus companheiros tenham tido várias parceiras sexuais (10). Além disso, o comportamento das mulheres com múltiplos parceiros é de maior risco para contrair DST e AIDS. O maior número de parceiros sexuais nos últimos três meses está entre os principais fatores de risco para DST ${ }^{(4)}$.

Os acadêmicos avaliados nesse estudo apresentaram dificuldade em questionar dados relacionados à sexualidade, visto que as maiores taxas de não registro foi para libido $(82,5 \%)$, hábito sexual (34\%) e dispareunia $(26,5 \%)$. A expressão da sexualidade humana é variável entre as diferentes sociedades, como são variáveis suas culturas e religiões. O que é normal para um povo pode não ser para outro. Uma mesma comunidade pode ter vários padrões de sexualidade, dependendo da história ou do nível social(10). É importante levar isso em consideração ao se questionar sobre a sexualidade de um indivíduo.

Dados relacionados à libido, orgasmo e dispareunia estão ligados diretamente à qualidade da relação sexual e devem ser questionados. Os problemas sexuais, geralmente permanecem ocultos por longo tempo, perturbando a vida do indivíduo e do casal. É difícil externar um distúrbio sexual com a mesma desenvoltura com que se comenta uma disfunção digestiva ou respiratória. Não se ouve as pessoas dizerem que não conseguem um orgasmo ou uma ereção ou que só obtêm prazer quando praticam sexo de uma maneira peculiar ou assumem tal posição para o coito $^{(10)}$. A dispareunia na mulher pode ter várias causas. Deve-se diferenciar se a dor é no início da penetração, no fundo da vagina ou se ocorre após a relação. Às vezes, depende da posição que o casal assume, da freqüência e do hábito das relações e do grau de desejo ${ }^{(10)}$.

o sangramento pós-coito, dado preenchido em $74,5 \%$ dos registros, é um dos sintomas que pode estar associado ao câncer de colo do útero na fase inicial da doença, quando ainda existe grandes perspectivas de cura $^{(1)} \mathrm{e}$, por isso deve ser questionado durante a consulta ginecológica.

\section{Tabagismo e etilismo}

Na maioria dos prontuários está registrado os hábitos relacionados ao tabagismo (79\%) e etilismo $(77,5 \%)$. O tabagismo é considerado pela Organização Mundial da Saúde (OMS) a principal causa de morte evitável em todo o mundo. A OMS estima que um terço da população mundial adulta, isto é, 1 bilhão e 200 
milhões de pessoas (entre as quais 200 milhões de mulheres), sejam fumantes. No Brasil, estima-se que cerca de 200.000 mortes/ano são decorrentes do tabagismo(19). O tabaco, em todas as suas formas, aumenta o risco de mortes prematuras e limitações físicas por doença coronariana, hipertensão arterial, acidente vascular encefálico, bronquite, enfisema e câncer. Entre os tipos de câncer relacionados ao uso do tabaco incluem-se os de pulmão, boca, laringe, faringe, esôfago, estômago, fígado, pâncreas, bexiga, rim e colo do útero(1). A mulher fumante tem um risco maior de infertilidade, câncer do colo do útero, menopausa precoce, em média dois anos antes, dismenorréia e irregularidades menstruais. Além desses riscos, as mulheres fumantes devem saber que o uso de anticoncepcionais associado ao cigarro aumenta em 10 vezes o risco de sofrer derrame cerebral e infarto ${ }^{(1)}$.

Em relação ao etilismo, estudos epidemiológicos têm demonstrado que o tipo de bebida (cerveja, vinho, cachaça) é indiferente, pois parece ser o etanol, propriamente, o agente agressor. Esta substância psicoativa tem a capacidade de produzir alteração no sistema nervoso central, podendo modificar o comportamento dos indivíduos que dela fazem uso. Por ter efeito prazeroso, induz à repetição e, assim, à dependência. Muitas doenças são causadas pelo uso contínuo do álcool: doenças neurais, mentais, musculares, hepáticas, gástricas, pancreáticas e entre elas o câncer. Isto sem falar nos problemas sociais que estão associados à ingestão de bebidas alcoólicas: acidentes de trânsito, homicídios, suicídios, faltas ao trabalho e atos de violência ${ }^{(19)}$.

\section{Exame físico}

O exame físico é de extrema relevância na avaliação do cliente e na definição do diagnóstico de Enfermagem, fornecendo subsídios para um planejamento da assistência de acordo com as necessidades e anormalidades encontradas. O exame físico deve ser completo, como em qualquer avaliação clínica eletiva. Especial atenção deve ser prestada ao abdômen (cicatrizes, ascite, irritação peritoneal), à pressão arterial, ao peso, à altura e a impressões gerais (estado geral, deambulação e postura, estado nutricional, etc.) Fazem parte da consulta ginecológica: o exame de mamas, da vulva e períneo, especular, toque vaginal bimanual e toque retal ${ }^{(10)}$.

A Tabela 4 mostra a distribuição dos itens realizados no exame físico na Casa de Parto.

Tabela 4: Distribuição dos registros de exame físico na Casa de Parto Natural. Fortaleza, 2006.

\begin{tabular}{|c|c|c|}
\hline VARIÁVEIS & $\mathbf{N}$ & $\%$ \\
\hline \multicolumn{3}{|l|}{ EXAME DAS MAMAS } \\
\hline Sim & 185 & 92,5 \\
\hline Não & 15 & 7,5 \\
\hline Incompleto & - & - \\
\hline \multicolumn{3}{|l|}{ VULVA E PERÍ NEO } \\
\hline Sim & 171 & 85,5 \\
\hline Não & 29 & 14,5 \\
\hline Incompleto & - & - \\
\hline \multicolumn{3}{|l|}{ EXAME ESPECULAR } \\
\hline Sim & 199 & 99,5 \\
\hline Não & 01 & 0,5 \\
\hline Incompleto & - & - \\
\hline \multicolumn{3}{|c|}{ I NSPEÇÃ́O VI SUAL COM ÁCI DO ACÉTI CO (I VA) } \\
\hline $\operatorname{Sim}$ & 145 & 72,5 \\
\hline Não & 55 & 27,5 \\
\hline Incompleto & - & - \\
\hline \multicolumn{3}{|l|}{ TESTE DE SCHI LLER } \\
\hline Sim & 182 & 91,0 \\
\hline Não & 18 & 9,0 \\
\hline \multirow{2}{*}{\multicolumn{3}{|c|}{ TOQUE BI MANUAL }} \\
\hline & & \\
\hline Sim & - & - \\
\hline Não & 200 & 100,0 \\
\hline Incompleto & - & - \\
\hline
\end{tabular}


Carvalho ALS, Nobre RNS, Leitão NMA, Vasconcelos CTM, Pinheiro AKB. Avaliação dos registros das consultas de enfermagem em ginecologia. Revista Eletrônica de Enfermagem [Internet]. 2008;10(2):472-483. Available from: http://www.fen.ufg. br/revista/v10/n2/v10n2a18.htm

Como pode ser observado, o Exame Clínico das Mamas (ECM), exame da vulva e períneo, exame especular, IVA e teste de Schiller foram registrados em mais de $70 \%$ dos prontuários. Por outro lado, o toque vaginal bimanual não foi descrito em $100 \%$ dos prontuários. Esse dado revela que há alguma dificuldade na realização ou no registro deste último exame, tanto por parte dos docentes que acompanham os acadêmicos como dos discentes, o que acaba deixando o exame da cliente incompleto.

No ECM podem ser identificadas alterações na mama e, se for indicado, serão realizados exames complementares. Este exame é realizado com a finalidade de detectar anormalidades na mama ou avaliar sintomas referidos por clientes e assim encontrar cânceres da mama palpáveis num estágio precoce de evolução. Aproximadamente $5 \%$ dos cânceres da mama são detectados por ECM em clientes com mamografia negativa, benigna ou provavelmente benigna. Durante a realização do exame é uma boa oportunidade para o profissional de saúde educar a população feminina sobre o câncer de mama, seus sintomas, fatores de risco, detecção precoce e sobre a composição e variabilidade da mama normal $^{(1)}$.

No exame da vulva e períneo, observamse a distribuição e as características dos pêlos, o trofismo vulvar, as lacerações e cicatrizes no períneo, a secreção exteriorizada, os condilomas e outras lesões cutâneas, a integridade ou não do hímen e o tamanho dos pequenos lábios e do clitóris ${ }^{(10)}$.

Através do exame especular, procura-se individualizar o colo uterino e avaliar o pregueamento e o trofismo da mucosa vaginal, secreções, lesões da mucosa, septações vaginais, condilomas, pólipos, cistos de retenção e ectopia ${ }^{(10)}$. Após a avaliação inicial do colo uterino e coleta da secreção vaginal para o exame à fresco, procede-se a aplicação do ácido acético à procura de lesões que foram realçadas pelo produto (mais brancas e brilhantes, ou leucoacéticas). Depois da inspeção visual com ácido acético (IVA), aplicase a solução de lugol para o teste de Schiller: se o colo cora de forma uniforme, escura, o teste é considerado normal; se, ao contrário, há áreas que não coram, o teste é considerado alterado $^{(10)}$.

\section{CONCLUSÕES}

Com este estudo percebe-se que os acadêmicos de enfermagem da instituição pesquisada estão realizando os registros de forma completa na maioria dos dados pesquisados. Embora uma parcela pequena dos dados tenha sido registrada em menos de $60 \%$ dos prontuários, o número de itens registrados em $100 \%$ deles é ainda menor.

Esses resultados revelam a necessidade de se investir tempo com os acadêmicos de enfermagem do serviço em questão sobre a importância de se registrar os dados de forma completa e correta, bem como trabalhar com docentes e discentes alguma deficiência existente, tendo como meta $100 \%$ de registro.

O registro dos dados do cliente no prontuário é imprescindível para uma assistência de qualidade, implementação adequada do processo de enfermagem, pesquisas, assim como fornece uma garantia legal para o profissional. Para tal, é necessário um maior empenho por parte dos docentes e discentes para realizar uma consulta de enfermagem de qualidade.

\section{REFERÊNCIAS}

1. Ministério da Saúde. Controle dos cânceres do colo do útero e da mama. Brasília (Brasil): Ministério da Saúde; 2006.

2. Ministério da Saúde; Secretária de Atenção à Saúde, Instituto Nacional do Câncer. Estimativas 2008: Incidência de Câncer no Brasil. Rio de Janeiro (Brasil): INCA, 2007.

3. Diógenes MAR, Rezende MDS, Passos NMG. Prevenção do câncer: atuação do enfermeiro na consulta de enfermagem ginecológica: aspectos éticos e legais da profissão. Fortaleza: Pourchain Ramos, 2001.

4. Secretaria de Saúde do Estado do Ceará. Saúde reprodutiva e sexual: um manual para a atenção primária e secundária (nível ambulatorial). Fortaleza (Brasil): SESA, 2002.

5. Gerk MAS, Barros SMO. Intervenções de enfermagem para os diagnósticos de enfermagem mais freqüentes em dois serviços 
Carvalho ALS, Nobre RNS, Leitão NMA, Vasconcelos CTM, Pinheiro AKB. Avaliação dos registros das consultas de enfermagem em ginecologia. Revista Eletrônica de Enfermagem [Internet]. 2008;10(2):472-483. Available from: http://www.fen.ufg. br/revista/v10/n2/v10n2a18.htm

públicos de assistência à saúde da mulher. Acta Paulista de Enfermagem. 2005; 18(3):260-8.

6. Iyer PW. Processo e diagnóstico em Enfermagem. Porto Alegre: Artes Médicas, 1993.

7. Ministério da Saúde. Prevenção do câncer do colo do útero. Manual Técnico. Organizando a Assistência. Brasília (Brasil): Ministério da Saúde; 2002.

8. Mussi NM. Técnicas Fundamentais de Enfermagem. São Paulo: Editora Atheneu, 1995.

9. Carrara HHA, Duarte G, Philbert PMP. Simpósio: Semiologia Especializada. Capítulo VIII Semiologia Ginecológica. Revista Medicina Ribeirão Preto. 1996;29(1):80-87.

10. Freitas $\mathrm{F}$, Menke $\mathrm{CH}$, Rivoire, WA, Passos EP. Rotinas em Ginecologia. 5a edição. Porto Alegre: Artmed, 2006.

11. Organización Mundial de la Salud. Control integral del cáncer cervicouterino: guía de práticas esenciales. Ginebra (Suiza): Organización Mundial de la Salud; 2007.

12. Santos MCL, Fernandes AFC, Cavalcante PP. Consulta ginecológica - Motivações e conhecimento da mulher sobre a prevenção do câncer do colo do útero. Revista da Rede de Enfermagem do Nordeste. 2004; 5(1):22-26.

13. Ministério da Saúde. Avaliação do Plano de Reorganização da Atenção à Hipertensão Arterial e ao Diabetes Mellitus no Brasil. Brasília (Brasil): Ministério da Saúde, 2004.

14. Ministério da Saúde. Hipertensão arterial sistêmica (HAS) e Diabetes mellitus (DM): protocolo. Brasília (Brasil): Ministério da Saúde, 2001.

15. Gutiérrez CA. Mulher na menopausa: declínio ou renovação? Rio de Janeiro: Rosa dos Tempos, 1992.

16. Lowdermilk DL, Perry SE, Bobak IM. O cuidado em enfermagem materna. 5a edição. Porto Alegre: Artmed, 2002.

17. Martins LBM. Fatores associados ao uso de preservativo masculino e ao conhecimento sobre DST/AIDS em adolescentes de escolas públicas e privadas do Município de São Paulo, Brasil. Cadernos de Saúde Pública. 2006;22(2): 315-23.

18. Paraguassú ALCB, Costa COM, Sobrinho CLN, Patel BN, Freitas JT, Araújo FPO. Saúde
Reprodutiva pré e pós-gestacional de adolescentes no Município de Feira de Santana, Bahia. Ciências e Saúde Coletiva. 2005; 10(2): 373: 80 .

19. Instituto Nacional do Câncer; Ministério da Saúde [Internet]. Rio de Janeiro: Ministério da Saúde (BR) [cited 2007 jan 17]. Estimativa 2006 - Incidência de Câncer no Brasil Avaliable from:

http://www.inca.gov.br/estimativa/2006/versao final.pdf

Artigo recebido em 20.06.07

Aprovado para publicação em 30.06.08 